\title{
ÉTUDE EXPÉRIMENTALE DE L'ECHINOCOCCOSE SECONDAIRE
}

\author{
Par A. DE WAELE et E. DE COOMAN
}

Matériel et méthode. - Le matériel parasitaire utilisé dans nos expériences est originaire du cheval. Dévé, qui, depuis 1901, s'est consacré l'étude de l'échinococcose secondaire, n'a trouvé que deux fois le parasite chez cet animal (1935) ; il utilisa surtout des larves développées chez le mouton, qui parait être l'hôte le plus fréquent en Normandie. Dans nos régions, la Flandre belge, l'échinococcose est rare chez cet animal : les hydatides recueillies pendant cinq années dans les abattoirs de la ville de Gand furent presqu'exclusivement trouvées dans le foie, et parfois dans le poumon, de chevaux (De Waele, 1935 ; De Cooman, 1937).

Dans ce vaste matériel, constitué par des milliers de vésicules, nous n'avons constaté aucun cas d'échinococcose alvéolaire : la larve prend, dans nos régions, surtout la forme uniloculaire, moins souvent la forme multiloculaire. D'autre part, la formation de vésicules filles est rare; nous ne l'avons observée que chez deux hôtes.

Au total, nos expériences ont porté sur 75 animaux; nous en faisons suivre la liste avec le mode d'infection.

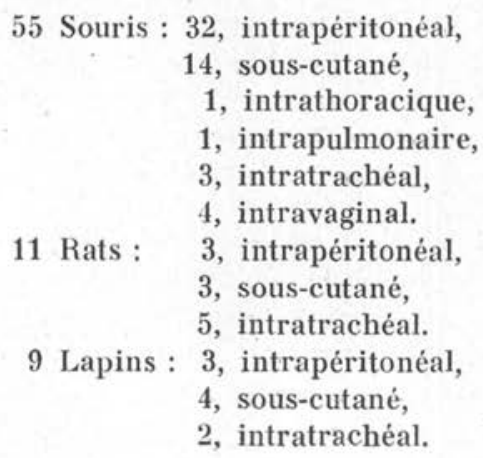

Pour l'injection intrapéritonéale, nous avons choisi la région de l'aine chez la souris et le rat, le flanc chez le lapin. L'inoculation sous-cutanée a été faite en divers endroits du corps. L'inoculation par la trachée se fit par injection directe de scolex dans l'organe

Annales de Parasitologie, T. XVI, $x^{\circ} 2 .-1^{\text {er }}$ mars 1938 , p. 121-132, 
mis à nu chez l'animal anesthésié, l'emploi d'une sonde ne fournissant pas de garanties suffisantes quant à la localisation exacte des parasites.

Vitalité et résistance des scolex. - Nos recherches ont été effectuées exclusivement avec du matériel parasitaire frais, et préalablement soumis à l'épreuve de vitalité ; celle-ci consiste dans l'observation sur la plaque de Pfeiffer, à une température de $37^{\circ}$ à $39^{\circ}$, de la mobilité et de l'évagination de scolex prélevés dans l'hydatide expérimentée.

Afin de pouvoir préparer des solutions artificielles correspondant au milieu naturel, nous avons déterminé, chez une trentaine d'hydatides, le $p \mathrm{H}$ du liquide : sa valeur, ramenée à $18^{\circ}$, varie entre 6,70 et 7,19 . Elle augmente chez des larves conservées dans la glacière à $5^{\circ}$, pour atteindre, après huit jours, 7,34. Ces valeurs sont inférieures à la moyenne trouvée par Coutelen (1927), qui probablement n'a pas fait la réduction de température. De toute façon, nous avons utilisé l'excellent ionomètre de Lautenschläger, qui fournit des données exactes à la seconde décimale près. Par ailleurs, il a été confirmé que le liquide hydatique infecté s'alcalinise plus rapidement.

Les scolex résistent à la congélation du liquide hydatique : extraits de vésicules conservées pendant 18 jours à des températures de $-2^{\circ}$ à $-4^{\circ}$, ils reprennent, après dégel du liquide, leur mobilité et s'évaginent à la température convenable.

\section{EXPÉRIENCES D'INOCULATION}

Afin de ne pas allonger cet exposé, nous avons établi, pour chaque espèce animale expérimentée, des tableaux résumant les résultats obtenus par les différents modes d'infection.

Discussion du tableau I. - Le matériel est constitué par des souris blanches, traitées par inoculation intrapéritonéale.

Abstraction faite des six premiers cas, où les animaux sont morts avant le début du phénomène de vésicularisation, nous avons obtenu un résultat positif chez toutes les souris : les 26 animaux étaient atteints d'échinococcose secondaire. Cette réceptivité cent pour cent de la souris blanche, pour des scolex d'hydatides de cheval, concorde avec les résultats obtenus par Dévé (1933, 1934) avec du matériel extrait de moutons.

La présence ou l'absence préalable de vésicules filles dans l'hydatide primaire n'a aucune influence sur le développement ultérieur 


\begin{tabular}{|c|c|}
\hline 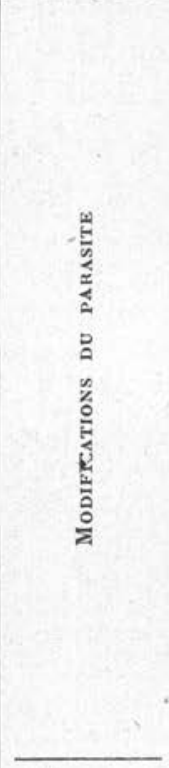 & 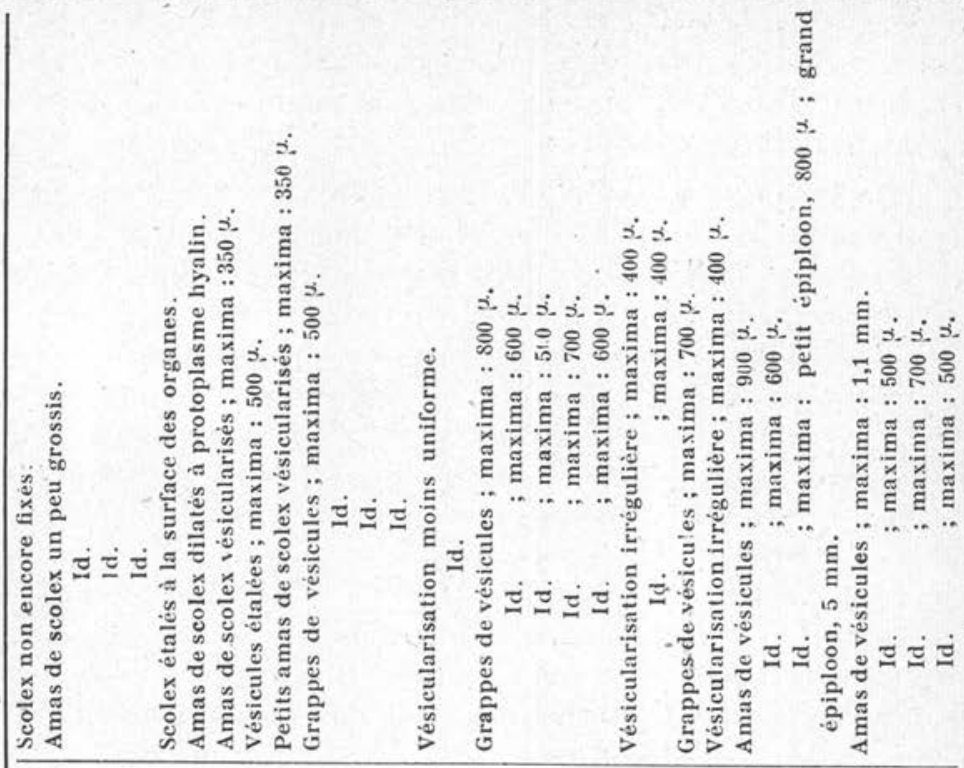 \\
\hline 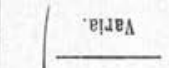 & 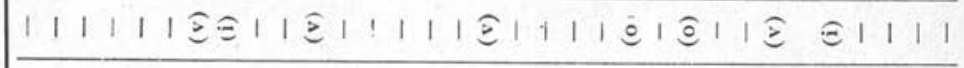 \\
\hline ค1еप & $\mid 1+1+++111111\lrcorner 1\lrcorner 1+1+11111+1$ 1 1111 \\
\hline 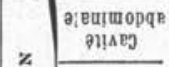 & $++++1111111++111111+11111111+++11+1$ \\
\hline 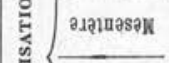 & $1111111+11+111111+11111+1++11111$ \\
\hline 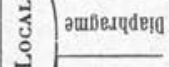 & $1111++1+1+1+11+111+1++++1+1 \quad 11111$ \\
\hline 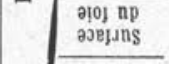 & $11+1+++++1+++1++1+1+++++1+1+++++$ \\
\hline $\begin{array}{c}\text { u o oddd } \\
\text { puestg } \\
\end{array}$ & $|1| 1|1| 1|1|++1|1| 1++++++++++++++1+++$ \\
\hline $\begin{array}{c}\text { uovoldida } \\
\text { ulad } \\
\end{array}$ & $|1| 1|1+1|+1+++++++++++++++1+++++++$ \\
\hline 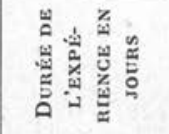 & 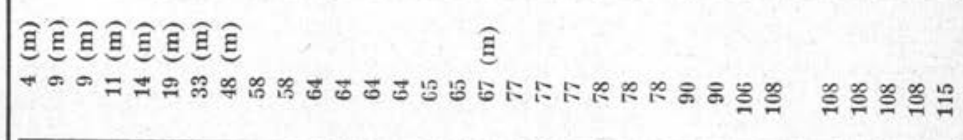 \\
\hline 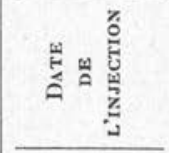 & 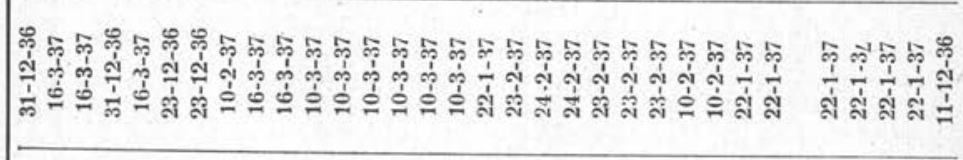 \\
\hline 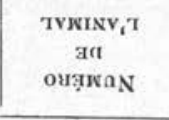 & 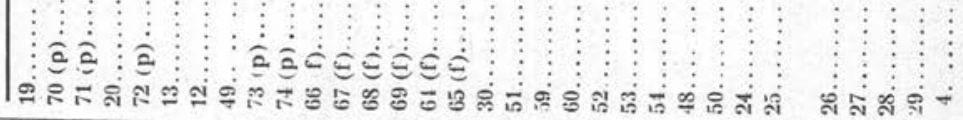 \\
\hline
\end{tabular}


des scolex : dans les deux cas, la vésicularisation se réalise d'une façon identique $\left(\mathrm{N}^{\circ} 64\right.$ à 69$)$. Nous en concluons que les hydatides encore dépourvues de ces formations représentent tout simplement un stade moins avancé, qui, dans des circonstances favorables, peut conduire à la formation de vésicules filles.

La nature de l'organe où s'est développée l'hydatide primaire n'influence guère la spécificité de l'organe où se développent ses scolex. Que la larve parasite soit extraite d'un foie ou d'un poumon de cheval, ses scolex ne se développent pas moins dans les organes les plus variés de la souris. Les localisations les plus fréquentes sont le petit et le grand épiploon, la surface du foie, la face inférieure du diaphragme ; le mésentère est moins souvent atteint et, quelquefois, on trouve les parasites, soit libres dans la cavité abdominale, soit fixés sur la rate, le processus vaginalis du mâle ou l'enveloppe péritonéale de l'oviducte chez la femelle. A la surface du foie, de la rate et du diaphragme, nous avons trouvé les vésicules étalées, formant des taches blanches; ailleurs, elles formaient surtout des grappes plus ou moins importantes.

Dans tous les cas observés, les scolex inoculés par injection intrapéritonéale ont été retrouvés dans la cavité abdominale. Nos expériences n'ont pas confirmé la thèse de Coutelen (1936), suivant laquelle les scolex pourraient, par migration active trans-diaphragmatique, passer dans la cavité thoracique. Cependant, nous ne considérons pas ce passage comme impossible; en effet, il sera démontré plus loin que les scolex sont capables de traverser du tissu musculaire. D'autres auteurs ont d'ailleurs observé le phénomène : Bacigalupo $(1933,1934)$ a obtenu, chez le lapin et le cobaye, une échinococcose secondaire du péritoine et du poumon par la voie digestive. Notons cependant que, ni Dévé (1933), ni Coutelen (1936), n'ont obtenu une confirmation de ces résultats.

D'autre part, deux fois les scolex ont été retrouvés à l'intẻrieur des tissus du foie ( $\mathrm{n}^{\circ} 26$ et 49 ). Cet organe avait réagi à l'invasion des parasites en les englobant dans une gaine de stroma fibroïde, autour de laquelle les tissus étaient d'ailleurs fortement altérés et infiltrés. Ceci confirme les observations de Coutelen (1936) qui, à l'encontre de Dévé (1936), a constaté également la migration de scolex dans le foie de la souris blanche.

Discussion du tableau II. - Le matériel était constitué par des souris blanches, traitées par inoculation sous-cutanée dans des régions différentes du corps. Sur les 14 animaux mis en expérience, 4 sont morts prématurément. Les 10 autres donnèrent 8 cas positifs de vésicularisation, soit 80 pour 100 . Ces résultats ne concordent pas 
avec les observations de Dévé (1934), qui, sur 18 expériences, obtint au total 11 résultats négatifs, soit un pourcentage de vésicularisation qui atteint à peine 38,7 . On peut se demander si ces divergences ne sont pas produites par la différence d'origine des hydatides

TABLEAU II

Souris. Inoculalion sous-culanée

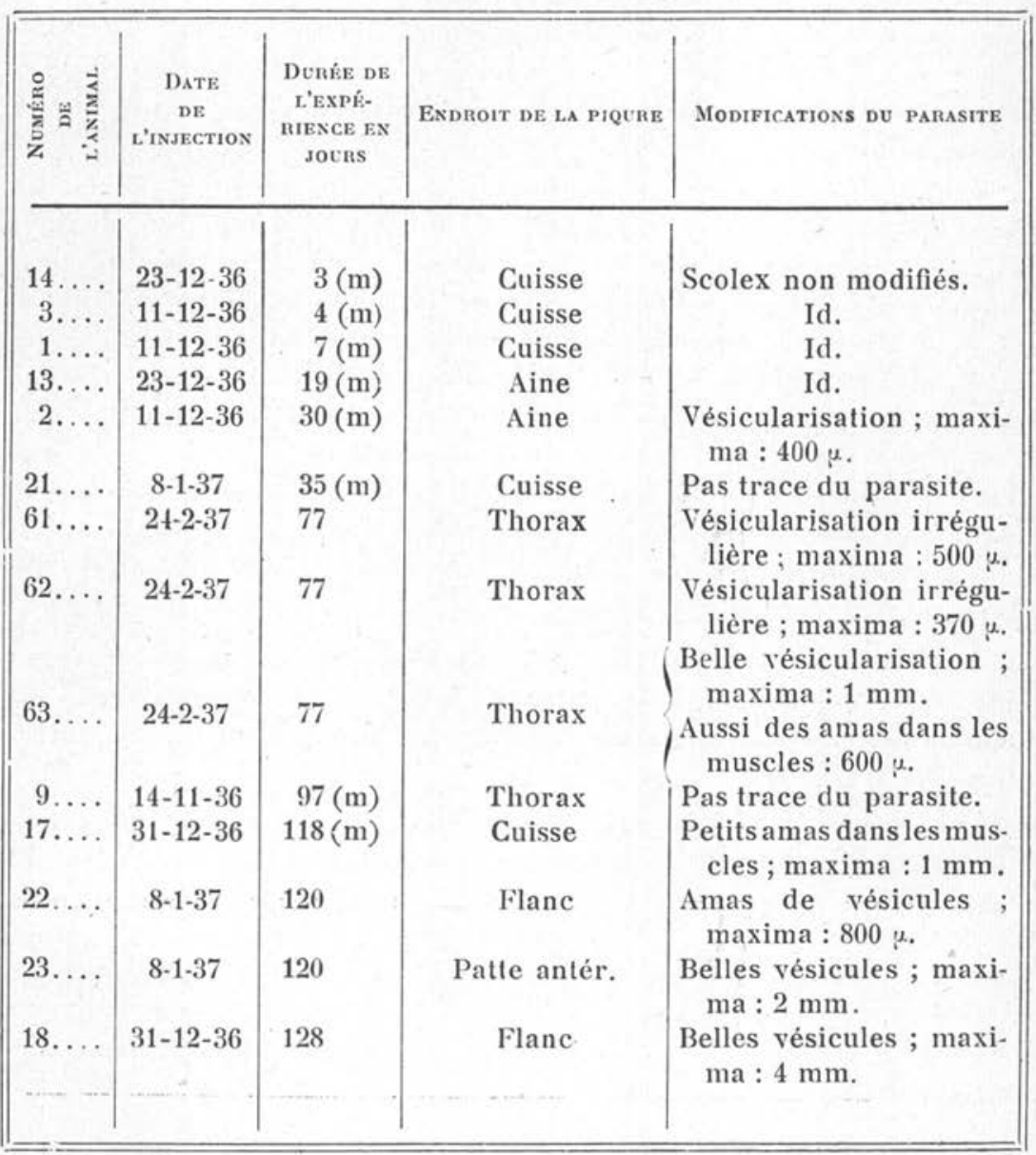

dont furent extraits les scolex. Sur le total des expériences de Dévé, dans 13 cas le matériel fut extrait d'hydatides de mouton et dans 5 cas d'hydatides de cheval. Il obtint 6 résultats positifs pour le mouton et 1 seul pour le cheval, soit respectivement un pourcentage 
de 42,6 et de 20,0. Ces considérations augmentent singulièrement la discordance entre nos résultats et ceux de Dévé. Il ne nous est pas possible, pour le moment, d'en fournir l'explication. De toute façon, nos recherches ont été effectuées avec du matériel frais et nous concluons à une grande réceptivité de la souris blanche à l'échinococcose secondaire, même si les scolex ont été introduits sous la peau.

L'évolution du processus sous-cutané de vésicularisation est en tout point comparable au développement dans la cavité abdominale : le gonflement et la croissance se font de la même façon et le parasite atteint à peu près les mêmes stades après une durée d'infection égale.

Deux fois, les scolex vésicularisés ont été retrouvés dans les muscles $\left(\mathrm{n}^{\circ} 17\right.$ et 63$)$. Les parasites sont, par conséquent, capables de traverser des territoires musculaires; c'est la raison pour laquelle nous ne rejetons pas, $\dot{a}$ priori, la thèse de Coutelen, suivant laquelle les scolex peuvent émigrer de la cavité abdominale à travers le diaphragme dans la cavité thoracique. Toutefois, nous n'avons pu constater nous-mêmes ce phénomène. Il est remarquable que l'évolution des scolex dans le tissu musculaire est plus lente que dans le tissu eonjonetif sous-cutané ; ce retard dans le développement doit, sans aucun doute, être attribué à une plus grande résistance mécanique des tissus qui entrave l'expansion vésiculaire du parasite.

Discussion du tableau III. - Les 4 souris infectées par le vagin ont donné des résultats négatifs. Ce petit nombre d'expériences est évidemment insuffisant pour conclure à l'impossibilité d'une infec-

TABlEAU III

Souris. Inoculation inlravaginale

\begin{tabular}{|c|c|c|c|}
\hline$\frac{2}{2}$ & $\begin{array}{c}\text { Date } \\
\text { DE } \\
\text { L'TNJECTION }\end{array}$ & $\begin{array}{l}\text { DURÉB } \\
\text { DE } \\
\text { L'EXPÉRIENCE } \\
\text { EN JOURS }\end{array}$ & Modifications dU PARASITE \\
\hline $\begin{array}{l}8 \ldots \\
7 \ldots \ldots \\
5 \ldots \\
6 \ldots\end{array}$ & $\begin{array}{l}12-12-36 \\
12-12-36 \\
12-12-36 \\
12-12-36\end{array}$ & $\begin{array}{r}30(\mathrm{~m}) \\
68(\mathrm{~m}) \\
147 \\
149\end{array}$ & $\begin{array}{l}\text { Pas de trace du parasite } \\
\text { Id. } \\
\text { Id. } \\
\text { Id. }\end{array}$ \\
\hline
\end{tabular}


tion expérimentale par cette voie. Elle est, sans aucun doute, difficile, car nombreuses sont les chances d'expulsion des scolex par suite de la sécrétion muqueuse et peut-être aussi des contractions musculaires, provoquées par l'introduction de la canule dans le tractus génital femelle. Dans les conditions naturelles, lorsqu'ils sont introduits par la copulation, les possibilités de développement des scolex sont certainement plus grandes; leur localisation sur le pénis reste toutefois problématique.

Discussion du tableau IV. - Dans aucun des cas où les scolex ont été introduits dans la trachée, nous n'avons trouvé de parasites dans le poumon. Cependant, cet organe constitue un excellent terrain de développement, puisque des scolex, injectés directement

Tableau IV

Souris. Inoculation inlralrachiale

\begin{tabular}{|c|c|c|c|}
\hline $\begin{array}{c}0 \\
\frac{a}{2} \\
\frac{d}{2} \\
z\end{array}$ & $\begin{array}{c}\text { DATB } \\
\text { DE } \\
\text { L'TNJECTION }\end{array}$ & $\begin{array}{c}\text { DURÉE } \\
\text { DE } \\
\text { L'EXPÉRIENCE } \\
\text { EN Jouns }\end{array}$ & Modifications de parasite \\
\hline $\begin{array}{l}43 \ldots \\
44 \ldots \\
31 \ldots\end{array}$ & $\begin{array}{l}9-2-37 \\
9-2-37 \\
6-2-37\end{array}$ & $\begin{array}{l}91 \\
94\end{array}$ & $\begin{array}{l}\text { Amas dans le tissu fibro-élastique } \\
\text { maxima }: 800 \mu \text {. } \\
\text { Amas dans le tissu fibro-élastique ; } \\
\text { maxima : } 800 ; . \text {. } \\
\text { Pas trace du parasite. }\end{array}$ \\
\hline
\end{tabular}

dans cet organe, s'y développent en grosses vésicules (tableau V). Il faut, par conséquent, admettre que les parasites introduits par injection intratrachéale n'ont pas émigré dans le poumon, mais au contraire furent expulsés avec le mucus. L'infection naturelle par voie trachéale s'avère peu probable. Toutefois, une restriction s'impose, étant donné le nombre peu important d'expériences faites à ce sujet.

Dans deux cas, une vésicularisation s'est produite dans le tissu fibro-élastique de la trachée, à l'endroit de l'inoculation ; ce développement doit être attribué à la localisation de scolex autour de l'ouverture produite par l'aiguille lors de l'injection.

Discussion du tableau v. - L'injection directe dans le poumon de la souris démontre que les scolex extraits d'hydatides de foie 
de cheval sont capables de se développer dans cet organe. Cette localisation, comme tant d'autres obtenues par l'injection intrapéritonéale, confirme le manque de spécificité des organes de la souris à l'égard de parasites extraits d'un organe déterminé d'un hôte d'espèce différente.

\section{TABLEAU V}

Souris. Inoculations diverses

\begin{tabular}{|c|c|c|c|c|}
\hline 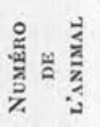 & $\begin{array}{c}\text { Date } \\
\text { DE } \\
\text { L'inJection }\end{array}$ & $\begin{array}{l}\text { DUREE DE } \\
\text { L'EXPÉ- } \\
\text { RIENCE EN } \\
\text { JOURS }\end{array}$ & ENDROIT DE LA PIQURE & ModificatioNs DU PARASITE \\
\hline $9 \ldots$ & $14-12-36$ & $97(\mathrm{~m})$ & Poumon & $\begin{array}{l}\text { Vésicularisation ; maxi- } \\
\text { ma }: 2 \mathrm{~mm} \text {. }\end{array}$ \\
\hline $76 \ldots$ & $22-12-36$ & 52, & Muscles pector. & $\begin{array}{l}\text { Vésicularisation peu } \\
\text { avancée. }\end{array}$ \\
\hline
\end{tabular}

L'inoculation dans les muscles du thorax constitue une confirmation du ralentissement observé dans le développement vésiculaire des scolex dans le tissu musculaire.

Discussion du tableau VI. - Ce tableau résume les expériences effectuées sur des lapins, par inoculation intrapéritonéale, sous-cutanée et intratrachéale. Tous les résultats furent négatifs : le parasite survit pendant quelques jours, puis, sous l'influence des sucs physiologiques de l'hôte, dépérit : ses tissus se disloquent $\left(\mathrm{N}^{\circ s} 37\right.$ et 39 ) et sont finalement résorbés $\left(\mathrm{N}^{\circ s} 34\right.$ et 36$)$.

Nos observations constituent une confirmation des résultats obtenus par Dévé : le lapin est réfractaire à l'inoculation de scolex d'hydatides de cheval, alors qu'il est réceptif pour les mêmes parasites du mouton. De ces expériences se dégage une conclusion intéressante: les scolex, nés dans des hydatides chez des hôtes spécifiquement différents, sont, il est vrai, morphologiquement semblables, mais différents cependant au point de vue physiologique. Ces transformations physiologiques s'extériorisent par une limitation dans la spécificité des hôtes où se fait leur développement ultérieur. Nous croyons que l'apparition de ces variations chez la larve constitue une phase initiale à la dissociation du ver sexué en espèces biologiques. 
TABLEAU VI

Lapins

\begin{tabular}{|c|c|c|c|c|}
\hline 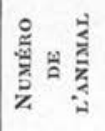 & $\begin{array}{c}\text { DATE } \\
\text { DE } \\
\text { L'INJECTION }\end{array}$ & $\begin{array}{c}\text { DURÉE } \\
\text { DE L'EXPÉ- } \\
\text { RIENCE } \\
\text { EN JOURS }\end{array}$ & $\begin{array}{c}\text { NATURE } \\
\text { DE L'INOCULATION }\end{array}$ & Modifications de PARASTte \\
\hline $32 \ldots$ & $6-2-37$ & $6(\mathrm{~m})$ & Intrapéritonéale & $\begin{array}{l}\text { Scolex non modifiés, } \\
\text { vivants. }\end{array}$ \\
\hline $33 \ldots$ & $6-2-37$ & $6(\mathrm{~m})$ & Id. & $\begin{array}{l}\text { Scolex non modifiés, } \\
\text { vivants. }\end{array}$ \\
\hline $34 \ldots$ & $6-2-37$ & 97 & Id. & Pas trace du parasite. \\
\hline $35 \ldots$ & $6-2-37$ & $3(\mathrm{~m})$ & Sous-cutanée & $\begin{array}{l}\text { Scolex non modifiés, } \\
\text { vivants. }\end{array}$ \\
\hline 37. & $6-2-37$ & $22(\mathrm{~m})$ & Id. & $\begin{array}{l}\text { Scolex morts, décompo- } \\
\text { sés. }\end{array}$ \\
\hline $39 \ldots$ & $6-2-37$ & $28(\mathrm{~m})$ & Id. & $\begin{array}{l}\text { Scolex morts, décompo- } \\
\text { sés. }\end{array}$ \\
\hline $36 \ldots$ & $6-2-37$ & 97 & Id. & Pas trace du parasite. \\
\hline $55 \ldots$ & $29-2-37$ & 80 & Intratrachéale & Pas trace du parasite. \\
\hline $38 \ldots$ & $6-2-37$ & 97 & Id. & Pas trace du parasite. \\
\hline
\end{tabular}

Discussion du tableau VII. - Les onze inoculations chez des rats ont fourni 3 résultats positifs. Subséquemment à une injection dans la trachée, des scolex, restés en dehors de cet organe, ont émigré sur le thymus et s'y sont développés. Cette localisation nouvelle démontre, une fois de plus, le manque de spécificité des organes à l'égard du parasite.

Dans un cas d'injection sous-cutanée $\left(\mathrm{N}^{\circ} 56\right)$, des scolex ont pénétré dans le tissu musculaire.

Il est remarquable que, sous aucune forme d'inoculation, le rat ordinaire n'ait fourni de résultat: il paraît être réfractaire. Le rat blanc, au contraire, est réceptif, tout au moins pour l'injection sous-cutanée. Ajoutons que l'animal présentant des vésicules sur le thymus était également un albinos.

Dévé a établi, d'autre part, que le rat blanc est réfractaire à l'inoculation sous-cutanée. Mais il ne faut pas perdre de vue que le matériel utilisé par cet auteur est originaire de moutons, alors que nous avons utilisé des parasites de chevaux. Ces expériences constituent, en somme, une confirmation de l'influence 
présumée de l'hôte sur l'hydatide, qui s'exprime par une différenciation biologique des scolex au moment de leur transformation en hydatide secondaire.

\section{Tableau VII}

Rats blancs (a) et ordinaires

\begin{tabular}{|c|c|c|c|c|}
\hline 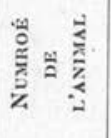 & $\begin{array}{c}\text { Date } \\
\text { DE } \\
\text { I.'iNJECTION }\end{array}$ & $\begin{array}{l}\text { DURÉE } \\
\text { DE L'EXPÉ- } \\
\text { nIENCE } \\
\text { EN JOURS }\end{array}$ & $\begin{array}{c}\text { Nature } \\
\text { De h'inocelation }\end{array}$ & Modifications dU PaRAstTE \\
\hline $40 \ldots$ & $9-2-37$ & 91 & Intrapéritonéale & Pas trace du parasite. \\
\hline $41 \ldots$ & $9-2-37$ & 91 & Id. & Id. \\
\hline 42 (a). & $9-2-37$ & 91 & Id. & Id. \\
\hline $58 \ldots$ & $23-2-37$ & $74(\mathrm{~m})$ & Sous-cutanée & $\begin{array}{l}\text { Scolex morts, décompo- } \\
\text { sés. }\end{array}$ \\
\hline 56 (a). & $23-2-37$ & 78 & Id. & $\begin{array}{l}\text { Amas de vésicules ; ma- } \\
\text { xima : } 800 \text {. } \\
\text { Amas de vésicules ; ma- } \\
\text { xima : } 1,1 \mathrm{~mm} \text {. }\end{array}$ \\
\hline 57 (a). & $23-2-37$ & 78 & Id. & $\begin{array}{l}\text { Aussi dans les muscles } \\
\text { thoraciques ; maxi- } \\
\text { ma: } 700 \mu .\end{array}$ \\
\hline $45 \ldots$ & $10-2-37$ & 91 & Intratrachéale & Pas trace du parasite. \\
\hline 46 (a). & $10-2-37$ & 91 & Id. & $\begin{array}{l}\text { Amas sur le thymus ; } \\
\text { maxima : } 600 \mu .\end{array}$ \\
\hline 47 (a). & $10-2-37$ & 91 & Id. & Pas trace du parasite. \\
\hline $16 \ldots$ & $31-12 \cdot 36$ & 128 & Id. & Id. \\
\hline $15 \ldots$ & $31-12-36$ & 130 & Id. & Id. \\
\hline
\end{tabular}

\section{Conclusions}

1. Dans la Flandre belge, l'hôte le plus fréquemment atteint d'échinococcose est le cheval. La larve prend, dans ces régions, surtout la forme uniloculaire, parfois multiloculaire, mais jamais la forme alvéolaire.

2. Dans le liquide d'hydatides fraìches, la valeur du $p \mathrm{H}$, réduite à $18^{\circ}$, oscille entre 6,70 et 7,19 . D'autre part, les scolex résistent pendant 18 jours à une température de $-2^{\circ}$ à $-4^{\circ} \mathrm{C}$.

3. Les hydatides de nos régions ne contiennent que rarement des vésicules filles. Cependant, il faut considérer comme des vésicules à l'état potentiel, les scolex des hydatides encore dépourvues de ces éléments. 
4. La souris blanche présente une réceptivité complète à l'injection intrapéritonéale de scolex d'hydatides de cheval. Ces observations concordent avec les résultats obtenus par Dévé avec des parasites originaires du mouton. Les organes de la souris ne présentent guère de spécificité à l'égard du matériel parasitaire qui s'est développé dans un organe déterminé de l'hôte intermédiaire.

Le siège de ces greffes échinococciques n'est pas exclusivement périviscéral : certaines vésicularisations se sont produites dans le foie, à l'intérieur des tissus. Cependant, nous n'avons pas observé de migrations à travers le diaphragme.

5. L'inoculation sous-cutanée a fourni, chez la souris blanche, un pourcentage élevé de résultats positifs, démontrant ainsi une grande réceptivité de cet animal pour cette forme d'inoculation effectuée aux dépens de scolex d'hydatides de cheval.

Dans certains cas, le parasite a émigré dans les muscles sousjacents ; il y subit une vésicularisation ralentie, mais normale.

6. L'inoculation par la trachée chez la souris, le rat et le lapin, par le vagin chez la souris, n'a pas fourni de résultats. L'opinion, émise par nous, suivant laquelle ces organes pourraient servir de voies naturelles à l'infection échinococcique, n’a, par conséquent, pas trouvé de confirmation expérimentale.

7. Le lapin est totalement réfractaire à l'inoculation de scolex d'hydatides de chevaux : les parasites sont tués après quelques jours et leurs tissus résorbés par les sucs physiologiques de l'hôte. Dévé, d'autre part, a établi la réceptivité de cet animal pour du matériel parasitaire extrait du mouton. Il n'est pas impossible que cette modification, apparue dans la spécificité de l'hôte inoculé, soit l'indice d'une phase initiale dans la dissociation de l'espèce systématique en espèces biologiques. L'influence physiologique de l'hôte intermédiaire, agissant d'une façon progressive, s'inscrirait dans le patrimoine héréditaire de l'hydatide, en modifiant le pouvoir d'adaptation de ses scolex. Ceux-ci, développés en ver sexué, produiraient des hydatides dont le terrain de développement se limiterait de génération en génération. Cette hypothèse fournit une explication plausible de la naissance de territoires où domine un hôte intermédiaire déterminé, tel le mouton en Normandie, le cheval dans la Flandre belge.

8. Le rat blanc est réceptif à l'inoculation sous-cutanée de scolex d'hydatides de cheval ; par contre, Dévé a montré que cet animal est réfractaire pour des parasites extraits de moutons. Ici également, les hôtes successifs ont laissé leur empreinte physiologique sur l'hydatide ; cette influence s'extériorise, lors de la vésicularisation, 
par une exaltation de la sensibilité du scolex à l'égard du milieu nouveau dans lequel il aboutit.

9. Le rat noir, à l'encontre de son congénère albinos, semble réfractaire à l'inoculation échinococcique.

\section{BibLIOGRAPHIE}

Bacigalupo (J.). - Quel est l'avenir du scolex échinococcique avalé par le lapin ? C.R. Soc. de Biol., CXIV, 1933, p. 89.

- L'échinococcose secondaire digestive chez le cobaye. C.R. Soc. de Biol., CXIV, 1933 , p. 390.

Coutelen (F.). - Essai de culture « in vitro » de scolex et d'hydatides échinococciques. Annales de parasitologie humaine et comparée, V, 1927, p. 7 .

- Migration active et sélective des scolex échinococciques dans le foie de la souris blanche au cours de certaines échinococcoses secondaires expérimentales. C.R. Soc. de Biol., CXXI, 1936, p. 730.

- Echinococcose secondaire expérimentale de la plèvre et du poumon de la souris blanche, par migration active transdiaphragmatique de scolex échinococciques inoculés dans la cavité péritonéale. C.R. Soc. de Biol., CXXI; 1936, p. 1266.

- Contrôle expérimental de certaines hypothèses récentes relatives au cycle évolutif du ténia échinocoque et à la prophylaxie de l'échirococcose. Rev. de méd. et d'hyg. tropic., 1936, p. 13.

De Cooman (E.). - Onderzoek over de ontwikkelingsmogelijkheden van de Echinococcuslarve, in verband met het probleem der secundaire échinococcose. Natuurvetensch. Tijdschr., XIX, 1937, p. 268.

Dévé (F.). - De l'échinococcose secondaire. Société d'éditions scientifiques, Paris, 1901.

- La souris blanche, animal réceptif pour les inoculations échinococciques. C.R. Soc. de Biol., CXIII, 1933, p. 1443.

- L'échinococcose secondaire expérimentale de la souris blanche. C.R. Soc. de Biol., CXIV, 1933, p. 445.

- Existe-t-il une échinococcose secondaire viscérale et singulièrement pulmonaire, à porte d'entrée digestive ? C.R. Soc. de Biol., CXIV, 1933, p. 669.

- L'échinococcose expérimentale de la souris blanche. Archivos internation. de la Hidatidosis, Montevideo, I, 1934, p. 85.

Réceptivité de la souris opposée à la résistance du lapin à l'inoculation de sable échinococcique du cheval. C.R. Soc. de Biol., CXIX, 1935, p. 351.

Sur le siège exclusivement infra-diaphragmatique et périviscéral des greffes échinococciques provoquées chez la souris blanche par inoculation intrapéritonéale de sable hydat:que. C.R. Soc. de Biol., CXXIII, 1936, p. 353.

De Waele (A.). - Recherches sur les migrations des Cestodes. Cinquième note : Etude de l'infection de l'hôte définitif par la larve hydatique. Bull. Acad. roy. de Belg., Classe des Sciences, 1935, p. 629.

Laboratorium voor algemeene Zoologie en Dierphysiologie der Rijksuniversiteit te Gent. (Directeur: Prof. A. De Waele) 